\title{
Damping Robot's Head Movements Affects Human-Robot Interaction
}

\author{
Guillaume Gibert, Florian Lance, Maxime Petit, Gregoire Pointeau, Peter Ford Dominey \\ INSERM U846 \\ Stem-Cell and Brain Research Institute \\ 69500 Bron, France \\ Université de Lyon \\ Université Lyon 1 \\ 69003 Lyon, France
}

\{guillaume.gibert, florian.lance, maxime.petit, gregoire.pointeau, peter.dominey\}@inserm.fr

\begin{abstract}
A new research platform has been developed to study humanrobot interaction and communication. In this setup, a humanoid robot is used as a proxy between two humans involved in dyadic interactions. An experimenter is bound with a humanoid robot. $\mathrm{He}$ can control in real-time and sensor free the eye and face/head movements performed by a humanoid robot with his own movements. The experimenter can perceive the scene as if he was the robot. Manipulations can be applied in real-time to any movement leaving the rest of the dynamics untouched. For instance, we have started investigating the effect of damping head movements during dyadic interaction. Preliminary results show that naive subjects' head nods increase when attenuation was applied on the robot's head movements.
\end{abstract}

\section{Categories and Subject Descriptors}

I.2.9 [Robotics]: Kinematics and dynamics, Manipulators, Sensors

\section{Keywords}

Wizard of Oz; teleoperation; interaction.

\section{INTRODUCTION}

Humanoid robots use advanced technologies and theories and are getting more and more complex. Yet, these social agents still fail to be as friendly and natural as a 'real' human in interaction. The main reason of this failure may be due to the extreme complexity of human multimodal communication. To study human-humanoid robot interaction, we developed an enhanced Wizard of $\mathrm{Oz}$ (super WoOZ) setup that mirrors some face, eye and head movements on a robot face rather than proposing a set of pre-defined responses as in the traditional WoOZ [1]. Hiroshi Ishiguro and colleagues developed a similar system with an android robot Geminoid HI-1 that can be remotely controlled by teleoperation. A confederate's lips and head movements are cloned on the robot face. The authors have started investigating how real human feel when interacting with this 'almost' human [2]. The main issue is

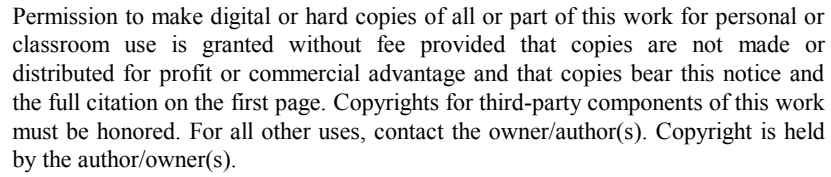
classroom use is granted without fee provided that copies are not made or distributed for profit or commercial advantage and that copies bear this notice and the full citation on the first page. Copyrights for third-party components of this work must be honored. For all other uses, contact the owner/author(s). Copyright is held by the author/owner(s).

HRI'14, March 3-6, 2014, Bielefeld, Germany.

ACM 978-1-4503-2658-2/14/03

http://dx.doi.org/10.1145/2559636.2563695 that the confederate perceives the scene as a $3^{\text {rd }}$ person. In our setup, a humanoid robot is used as a proxy between two humans involved in dyadic interactions. An experimenter is bound with a humanoid robot. He can control in real-time and sensor free the eye and face/head movements performed by a humanoid robot with his own movements. The experimenter can perceive the scene as if he was the robot.

We have started investigating the role of head movements during human-humanoid robot interaction with this super WoOZ platform. Our experiment followed the study proposed by [3]. In this study, confederates' head movements and facial expressions were manipulated in real-time during videoconference conversations by tracking them and reconstructing an avatar face. Results of this experiment show that increase in amplitude of head nods and lateral head turns in both naïve participant and confederate during dyadic interaction were noticed if attenuation was applied on the confederate's avatar head movements [3]. Our aim was to see if similar interaction loop effect can be created during human-humanoid robot interaction.

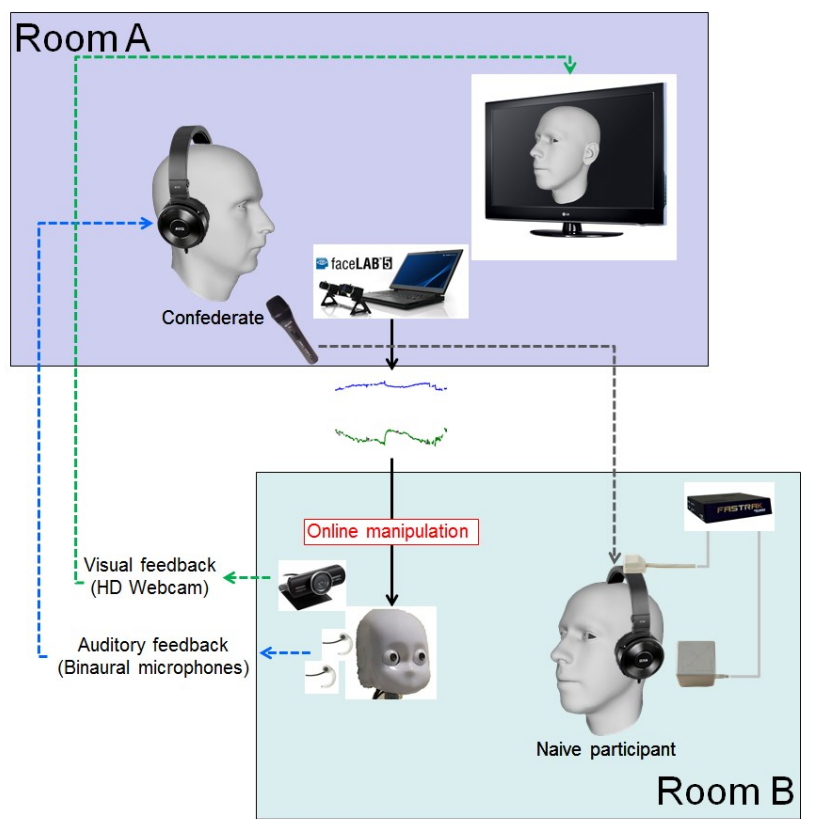

Figure 1: Schematic representation of the super Wizard of $\mathrm{Oz}$ platform. 


\section{METHOD}

\subsection{Setup}

The super $W o O Z$ platform consists of:

- $\quad$ a sensor (FaceLab5, Seeing Machines) to determine the confederate's face rigid and non-rigid motion and gaze position at any time;

- a software program to apply online manipulation to specific parameters;

- a humanoid robot (iCub [4]).

A schematic representation of the setup is shown in Figure 1. The FaceLab sensor (Seeing Machines) provides accurate measurement of the confederate's eye gaze and face/head motion. On the robot side, the iCub robot (http://www.icub.org/), a 1 metre high humanoid robot with eyes, head, arms and hands fully controllable in real-time. Between the sensor and the robot, a software program captures the gaze and face rigid and non-rigid motion data from the FaceLab sensor and applies (or not) modifications to the signals before sending it to the robot which mimics the confederate's gaze and head/face motion. The Facelab sensor provides the landmark positions on the face and more specifically on the lips and the eyebrows. This information is used to estimate the movements of the lip/jaw and the eyebrows which are then simulated on the robot face using a set of diodes. In addition, the eye closure was estimated using the FaceLab sensor and replicated on the robot eyelids. The naive participant wears headphones to hear the confederate's voice captured by a microphone remotely. A motion tracking sensor (Fastrak, Polhemus) is attached to the headphones to track his head movements. To bind the user into the robot, the user must sense the scene as if he was the robot. Auditory and visual feedbacks are provided to the experimenter using binaural microphones (MSTFB-2, The Sound Professionals, Inc.) and a High Definition (HD) webcam (Creative Live Cam Socialize HD).

\subsection{Procedure}

Three persons participated in the study. Each participant was instructed to freely interact with the iCub robot during 8 to 10 minutes. They were informed that the robot face/head and eye movements were controlled by the confederate's own movements. The robot voice was the confederate's voice. The confederate's head rotations were randomly attenuated by a factor 2 during 4 or 5 periods of 1 minute of interaction. The rest of the time the confederate's head rotations were replicated without modification on the robot. The head movements were recorded synchronously for the confederate and the naive subject at $60 \mathrm{~Hz}$.

\section{RESULTS}

Preliminary results show the same tendency as already reported in [3] for the naive subject (i.e., increase in head nods in the naive participant during attenuation compared to normal interaction) but not for the confederate. The temporal variation of the different agents' head nods is represented in Figure 2. The amplitude of subject's head nods (angular velocity) increases during attenuation periods (marked as " 0.5 ") compared to normal periods (marked as "1"). We are currently running this experiment on more subjects to assess if damping head movements in humanhumanoid robot interaction would create similar interaction loop effect as shown during human-human interaction.

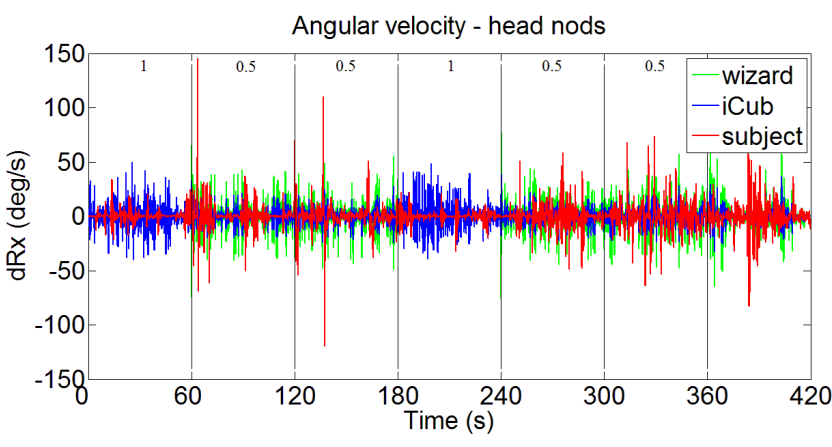

Figure 2: Temporal variation of the angular velocity of the wizard (green), the robot (blue) and the subject (red)'s head nods. During attenuation (0.5) of the robot head movements, increase in the subject's head nods is noticed.

\section{CONCLUSION}

A new research platform to study human-humanoid robot interaction has been presented. It has been used to study the role of head movements during dyadic interaction. As already reported for human-human interaction, damping head movements affects the interaction. In fact, naive subject interacting with a robot controlled in real-time by confederate's head movements increased their head movements when the robot's head movements were modified.

By manipulating specific movements without modifying the rest of the dynamics, the platform can be used to determine the acceptable limits for the human partner for various parametric manipulations. Several factors will be investigated independently of others: gaze latency, vergence, spatial shifting and head movements synchronization with gaze. Real acceptable limits will be determined by modifying parametrically specific behaviors. These limits may be used to create behavioral models for autonomous robots.

\section{ACKNOWLEDGMENTS}

This work was supported by the ANR SWoOZ project (11PDOC01901) and FP7 EFAA project (270490).

\section{REFERENCES}

[1] L. D. Riek, "Wizard of Oz Studies in HRI: A systematic Review and New Reporting Guidelines," Journal of Human-Robot Interaction vol. 1, pp. 119-136, 2012.

[2] I. Straub, S. Nishio, and H. Ishiguro, "Incorporated identity in interaction with a teleoperated android robot: A case study," in RO-MAN, 2010 IEEE, 2010, pp. 119124.

[3] S. M. Boker, J. F. Cohn, B. J. Theobald, I. Matthews, T. R. Brick, and J. R. Spies, "Effects of damping head movement and facial expression in dyadic conversation using real-time facial expression tracking and synthesized avatars," Philosophical Transactions of the Royal Society B-Biological Sciences, vol. 364, pp. 3485-3495, Dec 122009.

[4] G. Metta, G. Sandini, D. Vernon, L. Natale, and F. Nori, "The iCub humanoid robot: an open platform for research in embodied cognition," presented at the Proceedings of the 8th Workshop on Performance Metrics for Intelligent Systems, Gaithersburg, Maryland, 2008. 